\title{
Sleepless in Taiwan
}

\author{
Daniel L. Miller, MD
}

\author{
From WellStar Thoracic Surgery, WellStar Health System/Mayo Clinic Care Network, Marietta, Ga. \\ Disclosures: Author is on the Speaker Bureau for and receives honoraria from Acute Innovations, Inc. \\ Received for publication Aug 25, 2018; accepted for publication Aug 27, 2018. \\ Address for reprints: Daniel L. Miller, MD, WellStar Thoracic Surgery, 61 Whitcher St NW, Marietta, GA 30060 \\ (E-mail: daniel.miller@wellstar.org). \\ J Thorac Cardiovasc Surg 2019;157:781 \\ $0022-5223 / \$ 36.00$ \\ Copyright (c) 2018 by The American Association for Thoracic Surgery \\ https://doi.org/10.1016/j.jtcvs.2018.08.059
}

In this issue of the Journal, Cheng and colleagues ${ }^{1}$ report a pilot study evaluating the quality of sleep in adults who underwent surgical correction of pectus excavatum (PE) in Taiwan. Patients with PE defects have a poorer quality of life (QOL) related to both physiologic and psychological factors. The quality of sleep (QOS), which is a significant component of QOL, is usually not evaluated or even discussed in adults with pectus abnormalities. This study was the first to evaluate QOS and its effects on QOL in adult PE patients.

The patients (60) were divided into groups for analysis, 33 adults ( $>20$ years of age) were scheduled for Nuss procedure, whereas the control group was 30 normal adults who were similar in demographics of the pectus patients. All patients had to have no psychiatric conditions or mental illness, no illegal drug or narcotic use, and nonsmokers. All patients were evaluated extensively with several QOL questionnaires, which included a visual analog scale for pain and 5-item Brief Symptom Rating Scale, Beck Depression Inventory-II, and Short Form (36) version 2 surveys. QOS was accessed by the Pittsburgh Sleep Quality Index (PSQI), a subjective survey that is composed of 19 items with 7 components. Each PSQI item was rated on a scale from 0 to 3 ( $0=$ no difficulty, $3=$ extreme difficulty). The global sleep quality index was calculated with PSQI total scores, ranging from 0 to 21. Good QOS was a PSQI total score of 5 or less, whereas poor QOS was a PSQI total scores of at least 6. Objective measurement of QOS is best analyzed by polysomnography, which can also determine whether sleep apnea is the reason for poor QOS.

Patients with PE had poorer QOS and worse QOL than the control subjects. Of interest, poorer QOS was not related to severity of the PE or its physiologic factors but rather to psychological issues. After correction of the pectus defect by means of a Nuss procedure, QOS improved significantly.

Several studies have shown that psychological impact of $\mathrm{PE}$ is stronger than its physical impact in children and that psychological issues are correlated with severity of sternal impairment and age of the child. In Taiwan, the indication

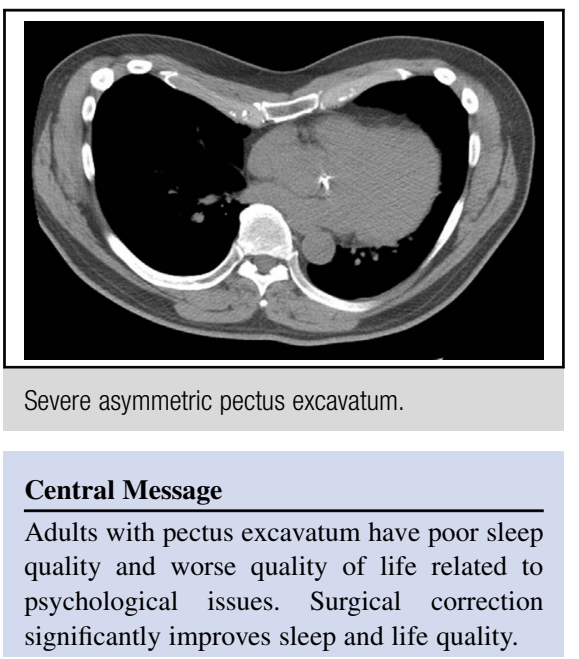

See Article page 769.

for a Nuss procedure in children is usually cosmetic, whereas, in the majority of adults, it is for physiologic factors.

This pilot study has set the framework for further evaluation of patients being considered for $\mathrm{PE}$ repair and QOL related to QOS. The evaluation of adults being considered for pectus surgery includes a 2-dimensional echocardiogram, pulmonary function tests, and 3-dimensional reconstruction computed tomographic scan and is subjected to final insurance approval in the United States. Adding QOL, especially QOS evaluation, may allow more patients with marginal physiologic data to be eligible to undergo PE correction. Administration of the QOL evaluation surveys, questionnaires and polysomnography is labor intensive, requiring significant personnel resources (full-time equivalents), and may only be possible in centers of excellence in the United States, especially in centers that perform Nuss procedures for adults. A US-based studied is warranted for validation. Patients who have undergone surgical treatment of PE are sleeping better in Taiwan; it is hoped that this will also be true for the future in the United States.

\section{Reference}

1. Cheng YL, Lan CC, Wu YK, Su WL, Yang MC. Poorer sleep quality among adult patients with pectus excavatum in Taiwan: a pilot study. J Thorac Cardiovasc Surg. 2019;157:769-80. 